\title{
P307
}

\section{Processing of Land 4D Seismic Data in Case of Limited Area of Repeated Survey - A Case Study from Otway Basin, Australia}

\author{
V. Shulakova* (CSIRO Petroleum), R. Pevzner (Curtin University of \\ Technology) \& M. Urosevic (Curtin University of Technology)
}

\section{SUMMARY}

Time lapse 3D seismic is an important part of monitoring and verification program of the Otway Project an Australian first demonstration of the deep geological storage of CO2 located on-shore in Victoria. From March, 2008 CO2-rich gas is being injected into a depleted gas reservoir at a depth of around 2050 metres. In year 2000 3D pre-production seismic data were acquired over large area, covering Naylor field, our $\mathrm{CO} 2$ sequestration site and also several adjacent small gas fields. Baseline 3D seismic data were acquired in December 2007, however due to logistical problems and cost of the survey size of the area was much smaller (only 3 sq. km). Processing of land time-lapse seismic surveys is a challenging task and in this case it was additionally complicated since limited migration aperture was determined to be one of main problems affecting imaging of the target horizon. To overcome this limitation we adopted an approach which is based on joint processing of two different 3D vintage sets with different size of survey area. 


\section{Amsterdam 'o9}

\section{Introduction}

Time lapse 3D seismic is an important part of monitoring and verification program of the Otway Project - an Australian first demonstration of the deep geological storage of $\mathrm{CO}_{2}$ located on-shore in Victoria. From March, $2008 \mathrm{CO}_{2}$-rich gas is being injected into a depleted gas reservoir at a depth of around 2050 metres. In year 2000 3D pre-production seismic data were acquired over large area, covering Naylor field, our CO2 sequestration site and also several adjacent small gas fields. Baseline 3D seismic data were acquired in December 2007, however due to logistical problems and cost of the survey size of the area was much smaller $(1.6 \times 1.9 \mathrm{~km})$. Repeated 3D survey will be acquired in January, 2009 with the same acquisition geometry.

Processing of land time-lapse seismic surveys is a challenging task and in this case it was additionally complicated since limited migration aperture was determined to be one of main problems affecting imaging of the target horizon. To overcome this limitation we adopted an approach which is based on joint processing of two different 3D vintage sets. This technique is demonstrated with pre-production (2000) and pre-injection (2007) seismic data sets recorded over Naylor sequestration site.

\section{Available seismic data}

The data available for this area consists of the following volumes: post stack migrated data acquired and processed for Santos in 2000, pre-stack data acquired in 2007 for Curtin university crew and VSP data was acquired in two boreholes by Schlumberger crew.

Surface seismic data were acquired with different parameters (Table 1), using different geometry (Figure 1) and hardware. Despite of large difference of the energy of the source the application of standard processing sequence to 2007 data produced reasonably good quality, comparable to 2000 dataset. The results are particularly comparable for the shallow horizons $(<1 \mathrm{~s})$ while deeper horizons (target horizon at $1.6 \mathrm{~s}$ ) data quality deteriorates for 2007 set due to week source (Figure 2). Additional problem as can be seen from Figure 1 relates to the small survey area of 2007 data set with respect to the depth of the target horizon $(2 \mathrm{~km})$. Because of that migration tails present a problem for the proper formation of the target image (Figure 2). Moreover, these artefacts will produce additional complications in cross-equalisation and differencing phase.

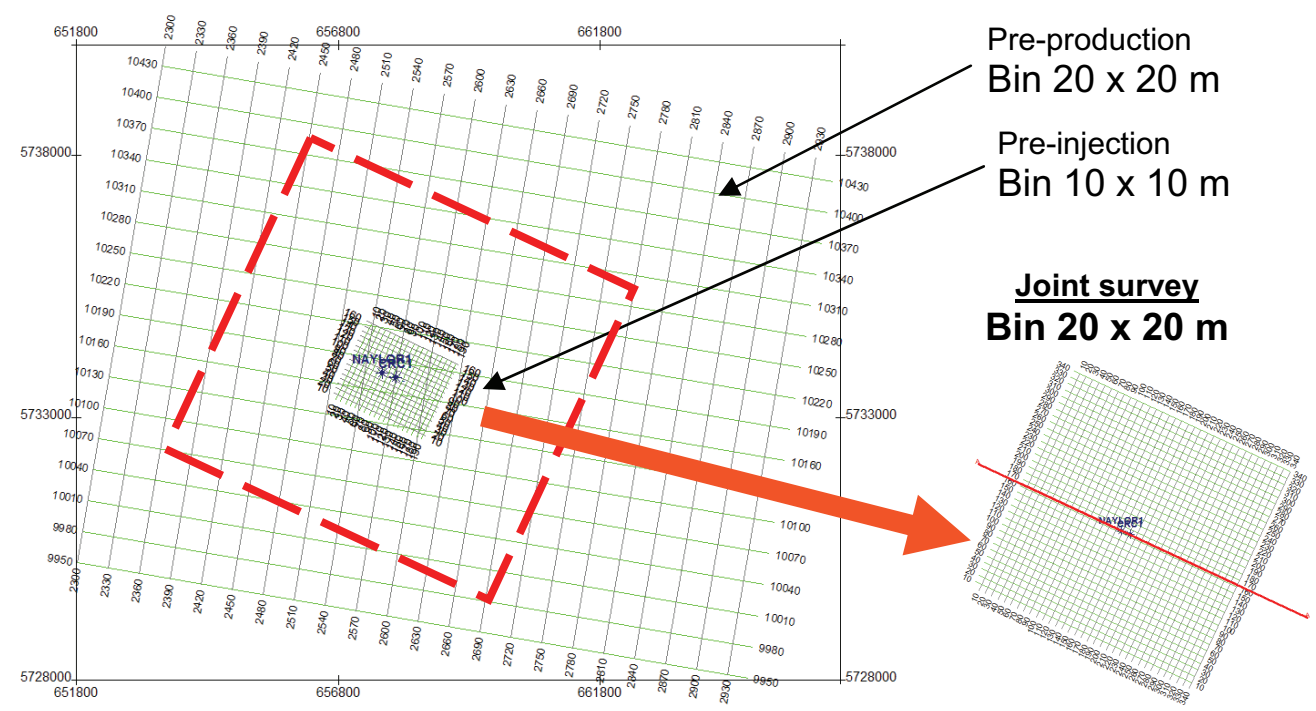

Figure 1: Basemap showing pre-production (bin $20 \times 20 \mathrm{~m}$ ), pre-injection (bin $10 \times 10 \mathrm{~m}$ ) surveys and new combined volume (bin $20 \times 20 \mathrm{~m}$ ). 


\section{Amsterdam 'og}

Table 1. Acquisition parameters.

\begin{tabular}{|c|c|c|}
\hline & Pre-production survey & Pre-injection survey \\
\hline Date & March 2000 & December 2007- January 2008 \\
\hline Bin size & $20 \times 20 \mathrm{~m}$ & $10 \times 10 \mathrm{~m}$ \\
\hline Source type & $\begin{array}{c}60000 \mathrm{lb} \text { HEMI Vibroseis, Sweep } \\
\text { frequency: } 5-90 \mathrm{~Hz}\end{array}$ & $\begin{array}{c}\text { Weight Drop (concrete breaker), } \\
750 \mathrm{~kg}, \text { free fall from } 1.2 \mathrm{~m}\end{array}$ \\
\hline Receiver type & Sensor SM4, $10 \mathrm{~Hz}, 375 \mathrm{ohm}$ & $10 \mathrm{~Hz}$ geophones \\
\hline Sample rate & $4 \mathrm{~ms}$ & $1 \mathrm{~ms}$ \\
\hline
\end{tabular}

\section{Reprocessing of repeated survey}

Main idea for reprocessing the small size 2007 survey was to increase the migration aperture by padding the volume with large size 2000 survey. To achieve this objective the following processing steps were utilized:

1. De- migration of pre-production cube

Unfortunately, only migrated pre-production data were available. So it was necessary to de-migrate it. We used phase shift time demigration. As no information about migration velocities used to produce cube was available, interval velocities were derived from VSP data, those were eventually averaged to a single velocity function was used for the entire area.

2. Interpolation of both cubes onto a common grid

Due to different acquisition geometries these two volumes had to be interpolated onto a common grid. For that purpose we used an algorithm developed by A. Khohlov in 2008 and implemented into processing software RadExPro (DECO Geophysical). As a result of this procedure we have two cubes sharing the same geometry $(20 \times 20 \mathrm{~m}$, Figure 1$)$.

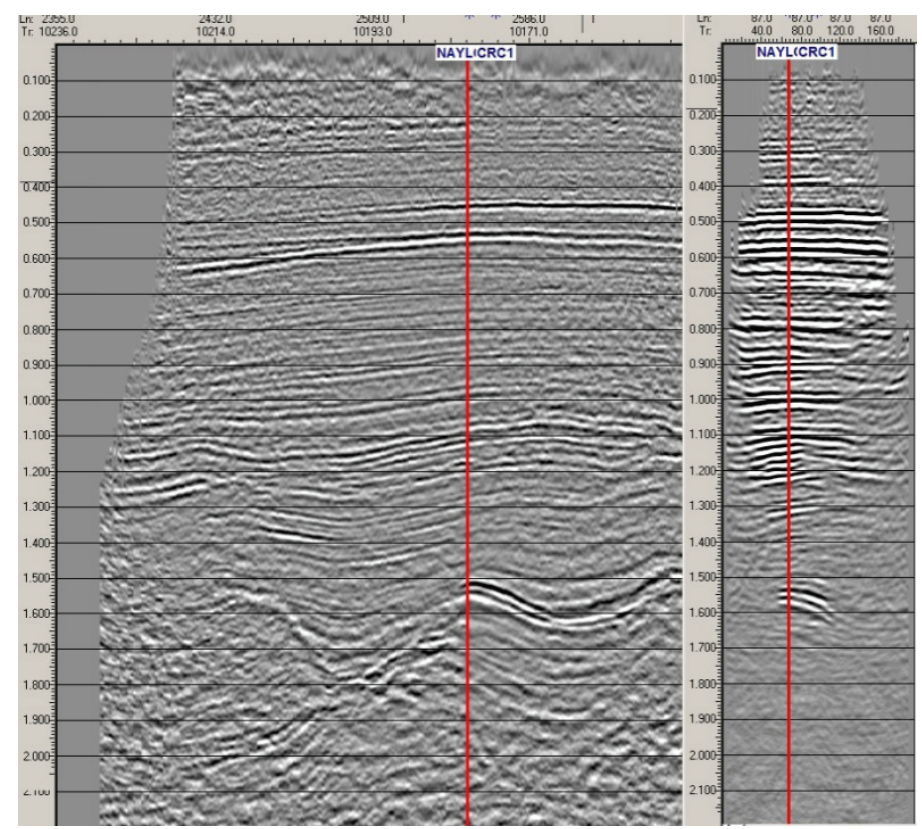

Figure 2: Comparison of two inlines extracted from migrated cubes: left is pre-production (2000) and right is pre-injection (2007-08).

\section{Time equalization of two datasets}

Different soil conditions (saturation of the soil) and seismic datum required time cross-equalization between these two datasets. Most of these time differences were in the long wavelength region. To calculate these time-shifts we used cross-correlation between corresponding traces from two cubes. We used cross-correlation window of $400 \mathrm{~ms}$. Calculations covered inline range - 124-214 and 


\section{Amsterdam 'og}

crossline range - 121-201. Correlation coefficient threshold of 0.6 was used which means that all traces that display lower correlation then that were excluded from analysis. Obtained shifts were interpolated to fill the entire grid and heavily smoothed across the entire grid. The averaged time shift between the two cubes is around $32 \mathrm{~ms}$ (Figure 3). This shift mainly corresponds to the difference in the seismic datum which was at sea level at 2000 data and $34 \mathrm{~m}$ for 2007.

\section{Amplitude balancing}

To cross-equalize the amplitudes between the two cubes we first compute amplitude envelope for each trace. The resultant envelopes were smoothed within $300 \mathrm{~ms}$ sliding window and corresponding traces from the two cubes were divided one into another to compute their ratio. The resultant functions then were smoothed by applying 2D spatial filter in two directions. Finally, to crossequalize amplitudes between two surveys, traces from pre-production cube were multiplied by these functions.

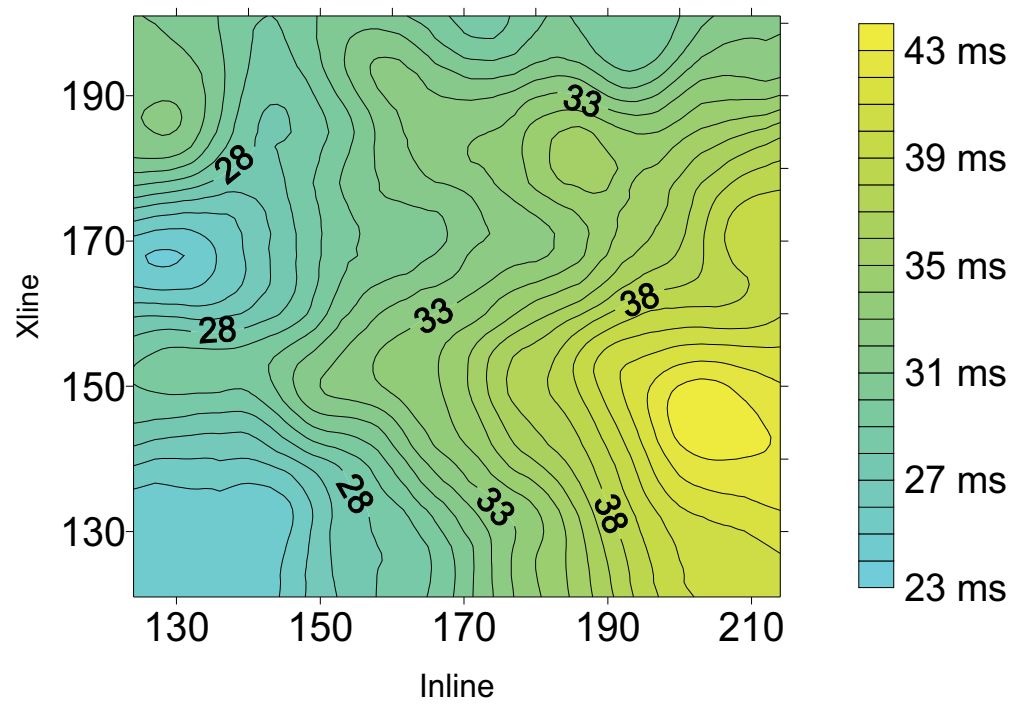

Figure 3: Smoothed time shifts.

$\underline{\text { 5. Matching filter design }}$

After time and amplitude equalisation a matching filter is calculated in Matlab with the following parameters: filter type - Wiener, symmetric with length of 100 samples, white noise level - $1 \%$, correlation coefficient threshold - 0.7. Filter design window was 400-1400 ms which excluded target horizon from the analysis.

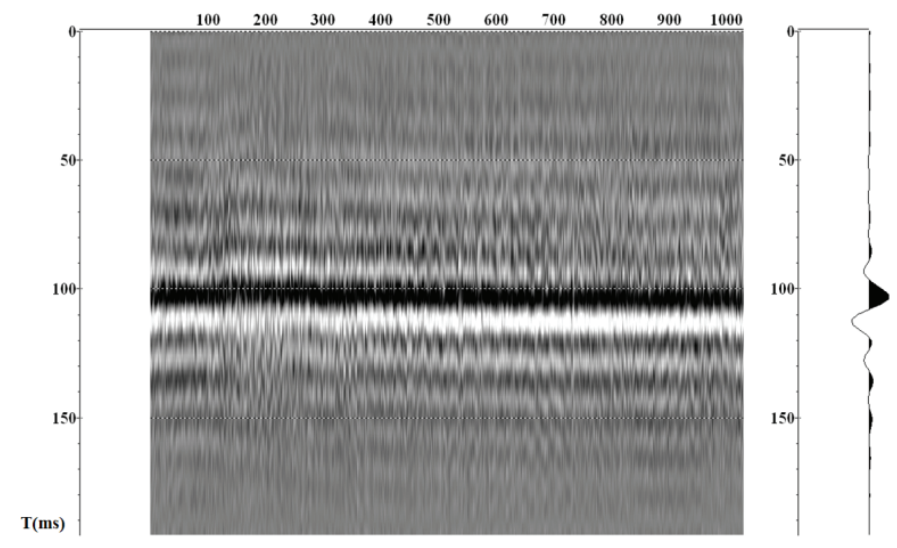

Figure 4: Filters for all valid traces. Average filter is shown on the right.

Filters were computed across large number of pairs of traces (threshold of 0.7). The final matching filter was then obtained by stacking all individually computed filters into a single one (Figure 4). This single filter was used to cross-equalize phase and amplitude spectra between two surveys. 


\section{D) \\ Amsterdam 'og}

6. Data merging and migration of the combined volume.

In the next step a new joint seismic volume was created with central part containing pre-injection dataset and outer part from pre-production data. The new combined cube was then migrated. Kirchhoff time migration was applied with RMS stacking velocities calculated from interval velocities used for the baseline data de-migration. The result of joint volume migration is shown in Figure $5 b$.

\section{Discussion}

We can make several observations from the results shown in Figure 5. Comparing the data in Figure $5 \mathrm{a}$ and $5 \mathrm{~b}$ we can say that these two completely different datasets were reasonably cross-equalized. Secondly there is an obvious improvement of a traceability of the target reflector (compare Figures $5 \mathrm{~b}$ and 5c). Finally it is clear that the joint cube is reasonably consistent with pre-production cube.

a)

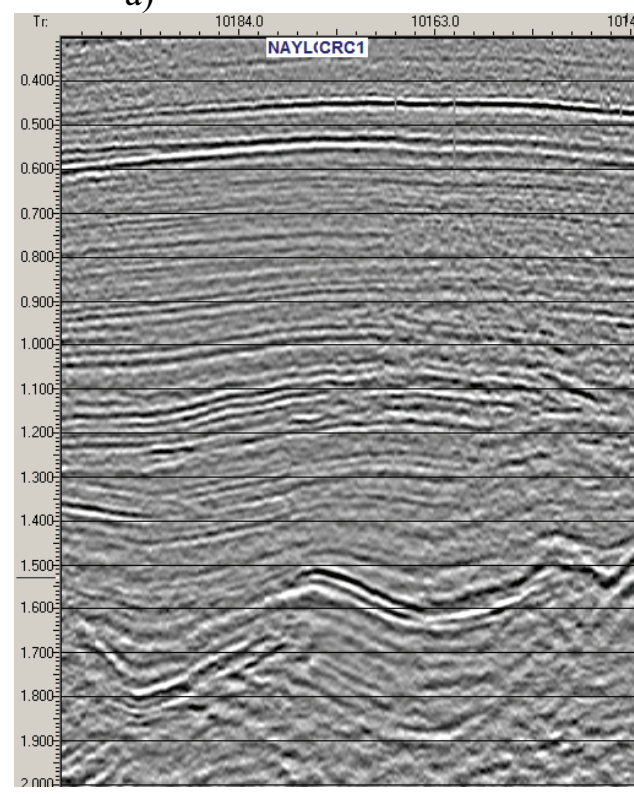

b)

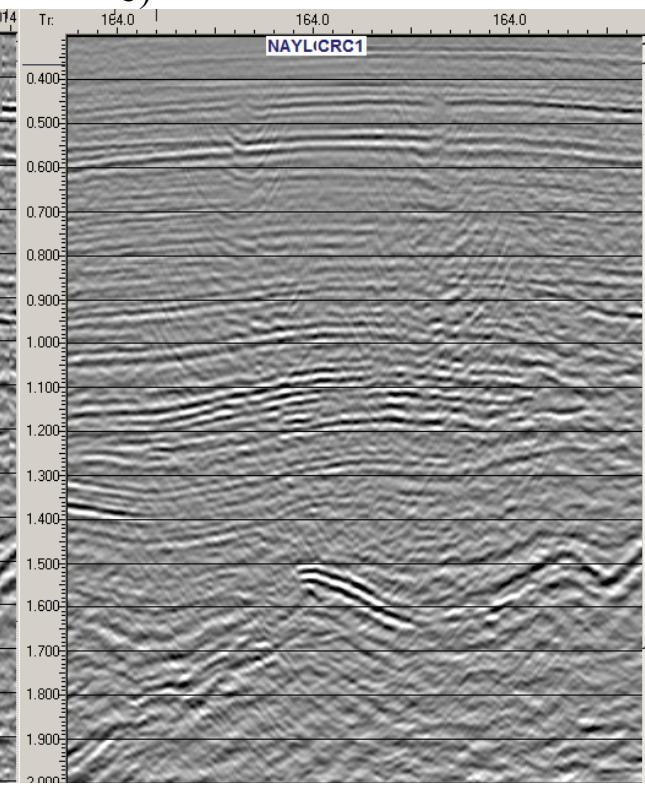

c)

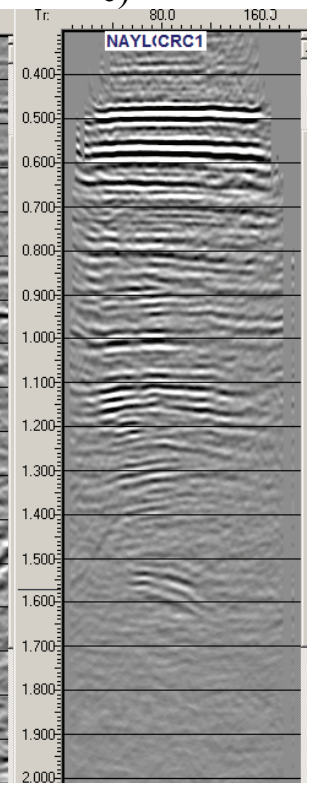

Figure 5: Inlines extracted from: a) pre-production cube, b) jointly migrated cube and c) preinjection cube.

We can say that the quality of migration image (Figure 5b) has improved significantly despite obvious problem of two datasets being quite dissimilar geometry, energy load and frequency content.

\section{Conclusions}

An approach to time-lapse data processing which can be applied in cases of insufficient migration aperture of repeated survey is suggested. It requires cross-equalization between surveys at processing stage and reduces migration artefacts. This approach was successfully tested on two different 3D data sets recorded in Otway basin, Australia in 2000 (pre-production) and 2007 (pre-injection). This approach can also be adopted for processing of time-lapse surveys recorded for monitoring of $\mathrm{CO}_{2}$ injection of this site.

\section{Acknowledgment}

We would like to thank $\mathrm{CO} 2 \mathrm{CRC}$ for funding this research project.

\section{References}

Final Report [2000]. Seismic Data Processing. OCV00 CURDIE VALE 3D, CGG Perth Processing Centre. 